PROCEEDINGS OF THE

AMERICAN MATHEMATICAL SOCIETY

Volume 140, Number 6, June 2012, Pages 1881-1896

S 0002-9939(2011)11061-X

Article electronically published on October 12, 2011

\title{
ZETA DETERMINANT FOR DOUBLE SEQUENCES OF SPECTRAL TYPE
}

\author{
M. SPREAFICO
}

(Communicated by Walter Van Assche)

\begin{abstract}
We study the spectral functions, and in particular the zeta function, associated to a class of sequences of complex numbers, called of spectral type. We investigate the decomposability of the zeta function associated to a double sequence with respect to some simple sequence, and we provide a technique for obtaining the first terms in the Laurent expansion at zero of the zeta function associated to a double sequence.
\end{abstract}

\section{INTRODUCTION}

Let $S=\{\lambda\}$ be a sequence of complex numbers. Under some mild requirements on $S$, it is possible to define the zeta function associated to $S$ by the Dirichlet series

$$
\zeta(s, S)=\sum_{\lambda \in S}^{\infty} \lambda^{-s}
$$

where $s$ is a complex variable, and the series in equation (11) converges in some domain of the complex plane. For example, if $S=\mathbb{N}_{0}=\{1,2, \ldots\}$ are the positive integers, then $\zeta(s, S)=\zeta_{R}(s)$ is the Riemann zeta function, and if $S$ are the norms of the integral ideals of a number field $\mathbb{K}$, then $\zeta(s, S)=\zeta_{\mathbb{K}}(s)$ is the Dedekind zeta function of $\mathbb{K}$; in both cases the series in equation (1) converges if $\operatorname{Re}(s)>1$. If $S$ is the spectrum of the Laplace operator on a compact Riemannian manifold of dimension $m$, then the series in equation (11) converges if $\operatorname{Re}(s)>\frac{m}{2}$.

Several authors studied analytic properties of spectral functions and regularized products associated to some classes of abstract sequences [17, 2, 10, 5, 6, and indeed the basic facts of this theory are contained in the classical works on Dirichlet series (see for example [4]). In particular, a general theory has been introduced and described in detail in the works of Jorgenson and Lang [5, 6, 7, 8]. Our works proceed along this line of investigation, but are more focused on regularized products, and in particular to results on the expansion of the zeta function at zero. It is well known that the determination of the value of the derivative of the zeta function at zero is a major problem in various areas of mathematics and theoretical physics. In number theory, it is related with the ideal class number, while in analysis and geometry it is used in order to define the functional determinant of a linear operator. With this aim, we introduced in [14 a class of abstract sequences of complex numbers, which we called of spectral type, that is a subclass

Received by the editors July 1, 2010 and, in revised form, February 2, 2011.

2010 Mathematics Subject Classification. Primary 11M41; Secondary 33E20.

(C)2011 American Mathematical Society 1881

Reverts to public domain 28 years from publication 
of the more general type introduced in [5], and we investigated the properties of the spectral functions associated to the sequences of that class. In a series of works [12, 13, 15, 16], we studied various different applications of the approach introduced in 14. In particular, in 13 and 15, some double sequences appeared, and we introduced some techniques that allowed us to tackle the expansion at zero of the associated zeta functions. These techniques permitted us to obtain very useful information in the different problems analyzed in those works. This motivates the investigation of a general purpose technique that permits us to deal with a large class of double or even multiple sequences. This is the aim of the present work, which has two sections. In Section 2, we review the definition of sequences of spectral type given in 14, and we give some further properties of the zeta function associated to the sequences of this type. In Section 3 we consider double (or multiple) sequences. We define a criterium for decomposing a double sequence with respect to some simple sequence of spectral type (Definition 3.1), and we prove our main result in Theorem 3.9 , where we give the expansion at zero of the zeta function associated to a decomposable double sequence.

\section{ZETA INVARIANTS FOR SEQUENCES OF SPECTRAL TYPE}

In this section, we recall from [14 the definition of sequences of spectral type, and we give some new results on the spectral functions associated to that class of sequences. Let $S=\left\{\lambda_{n}\right\}_{n=1}^{\infty}$ be a sequence of nonvanishing complex numbers with unique accumulation point at infinity. We order $S$ as $0<\left|\lambda_{1}\right| \leq\left|\lambda_{2}\right| \leq \ldots$ (if we need to include the number zero, we use the notation $\lambda_{0}=0$ ). The positive number

$$
\mathrm{e}=\mathrm{e}(S)=\limsup _{n \rightarrow \infty} \frac{\log n}{\log \left|\lambda_{n}\right|}
$$

is called the exponent of convergence of $S$. We are only interested in sequences with $\mathrm{e}(S)<\infty$. If this is the case, then there exists a least integer $p$ such that the series $\sum_{n=1}^{\infty} \lambda_{n}^{-p-1}$ converges absolutely. We assume $\mathrm{e}-1<p \leq \mathrm{e}$, and hence we have $p=[\mathrm{e}]$ (the integer part). The integer $p$ is called the genus of the sequence $S$, and we use the notation $\mathrm{g}=\mathrm{g}(S)=p$. The Weierstrass canonical product

$$
p(z, S)=\prod_{n=1}^{\infty}\left(1+\frac{z}{\lambda_{n}}\right) \mathrm{e}^{\sum_{j=1}^{\mathrm{g}(S)} \frac{(-1)^{j}}{j} \frac{z^{j}}{\lambda_{n}^{j}}}
$$

converges uniformly and absolutely in any bounded closed region of the complex plane and is an integral function of order $\mathrm{g}(S)$ which vanishes if and only if $z=-\lambda_{n}$ for some $n$. We call the open subset $\rho(S)=\mathbb{C}-S$ of the complex plane the resolvent set of $S$. We define the Gamma function associated to $S$ by the canonical product $\Gamma(z, S)=\frac{1}{p(z, S)}$. For further convenience, we use the variable $\lambda=-z$ for the Gamma function, with the convention that the variable $-\lambda$ is purely real on the negative part of the real axes. When necessary, in order to define the branch of analyticity of a meromorphic function, the domain for $\lambda$ will be some open subset of $\mathbb{C}-[0, \infty)$ in the complex plane. With this notation, for all $\lambda \in \rho(S)$, the Gamma function is

$$
\frac{1}{\Gamma(-\lambda, S)}=\prod_{n=1}^{\infty}\left(1+\frac{-\lambda}{\lambda_{n}}\right) \mathrm{e}^{\sum_{j=1}^{\mathrm{g}(S)} \frac{(-1)^{j}}{j} \frac{(-\lambda)^{j}}{\lambda_{n}^{j}}}
$$


We now introduce the definition of sequences of spectral type. Conditions for a sequence to be of spectral type can be found in [5] or [14].

Definition 2.1. Let $S=\left\{\lambda_{n}\right\}_{n=1}^{\infty}$ be a sequence of nonvanishing complex numbers with unique accumulation point at infinity and exponent of convergence e $(S)<\infty$. We call $S$ a sequence of spectral type (S-type) if the following conditions hold:

(1) there exist $c>0$ and $0<\theta<\pi$, such that the sequence $S$ is contained in the interior of the sector $\Sigma_{\theta, c}=\left\{z \in \mathbb{C}|| \arg (z-c) \mid \leq \frac{\theta}{2}\right\}$;

(2) the logarithm of the associated Gamma function has an asymptotic expansion for large $\lambda \in A D_{\theta, c}(S)=\mathbb{C}-\Sigma_{\theta, c}$ of the form

$$
\log \Gamma(-\lambda, S)=\sum_{\alpha} \sum_{k=0}^{K_{\alpha}} a_{\alpha, k}(-\lambda)^{\alpha} \log ^{k}(-\lambda)+o\left((-\lambda)^{\alpha_{N}}\right)
$$

where $\{\alpha\}$ is a decreasing sequence of real numbers $\alpha_{0}>\alpha_{1}>\cdots>\alpha_{N} \geq$ $-\infty$, and $k=0,1, \ldots, K_{\alpha} \in \mathbb{N}$, for each $\alpha$.

If $N$ is finite, we call the number $\alpha_{N}$ the order of the sequence $S$, and we use the notation o $(S)$. We say that $S$ has infinite order if $N$ is not finite, and we write $\circ(S)=-\infty$. We call the open set $A D_{\theta, c}(S)$ the asymptotic domain of $S$.

Remark 2.2. The point $\lambda=0$ belongs by definition to the domain of analyticity of $\Gamma^{-1}(-\lambda, S)$ for a sequence of spectral type, and $\log \Gamma(-\lambda, S)$ has a zero of order $\mathrm{g}(S)+1$ at $\lambda=0$.

We define some further spectral functions associated to a sequence $S$ of spectral type (see for example [5], p. 1): the zeta function, defined by the uniformly convergent series

$$
\zeta(s, S)=\sum_{n=1}^{\infty} \lambda_{n}^{-s},
$$

for $\operatorname{Re}(s)>\mathrm{e}(S)$, and by analytic continuation elsewhere, the heat function $(t>0)$

$$
f(t, S)=1+\sum_{n=1}^{\infty} \mathrm{e}^{-t \lambda_{n}}
$$

and, for each nonnegative integer $k$, and $\lambda \in \rho(S)$, the functions

$$
r_{k}(\lambda, S)=-\frac{d^{k+1}}{d \lambda^{k+1}} \log \Gamma(-\lambda, S)=k ! \sum_{n=1}^{\infty}\left(\frac{(-1)^{k}}{\left(\lambda-\lambda_{n}\right)^{k+1}}+\sum_{j=0}^{\mathrm{g}(S)-k-1}\left(\begin{array}{c}
j+k \\
j
\end{array}\right) \frac{\lambda^{j}}{\lambda_{n}^{j+k+1}}\right) .
$$

These spectral functions are clearly strictly related to each other (under some general regularity assumptions). In particular, the following complex integral representations hold (see [12] and [14, Proposition 2.4). Here we use the notation $\Sigma_{\theta, c}=$ $\left\{z \in \mathbb{C}|| \arg (z-c) \mid \leq \frac{\theta}{2}\right\}$, with $c \geq \delta>0,0<\theta<\pi$. We use $A D_{\theta, c}=\mathbb{C}-\Sigma_{\theta, c}$ for the complementary (open) domain and $\Lambda_{\theta, c}=\partial \Sigma_{\theta, c}=\left\{z \in \mathbb{C}|| \arg (z-c) \mid=\frac{\theta}{2}\right\}$, oriented counterclockwise, for the boundary. 
Lemma 2.3. For $\operatorname{Re}(s)>\mathrm{e}(S)$,

$$
\begin{aligned}
f(t, S)-1 & =\frac{1}{2 \pi i} \int_{\Lambda_{\theta, c}} \mathrm{e}^{-\lambda t} r_{0}(\lambda, S) d \lambda \\
& =-\frac{t}{2 \pi i} \int_{\Lambda_{\theta, c}} \mathrm{e}^{-\lambda t} \log \Gamma(-\lambda, S) d \lambda, \\
\zeta(s, S) & =\frac{1}{\Gamma(s)} \int_{0}^{\infty} t^{s-1}(f(t, S)-1) d t \\
& =\frac{s}{\Gamma(s)} \int_{0}^{\infty} t^{s-1} \frac{1}{2 \pi i} \int_{\Lambda_{\theta, c}} \frac{\mathrm{e}^{-\lambda t}}{-\lambda} \log \Gamma(-\lambda, S) d \lambda d t .
\end{aligned}
$$

Lemma 2.4. If $k=\mathrm{g}=\mathrm{g}(S)$, then

$$
r_{\mathrm{g}}(\lambda, S)=-\mathrm{g} ! \zeta(\mathrm{g}+1, S-\lambda)=-\mathrm{g} ! \sum_{n=1}^{\infty}\left(\lambda_{n}-\lambda\right)^{-\mathrm{g}-1} .
$$

This is a uniformly convergent series, and we can take the limit for $\lambda \rightarrow \infty$ that is 0 . This implies that $r(\lambda, S)$ cannot have a term such as $(-\lambda)^{m}$ and consequently $\log \Gamma(-\lambda, S)$ cannot have a term such as $(-\lambda)^{m+1}$, with $m \geq \mathrm{g}$.

Lemma 2.5. Let $S$ be a sequence with finite exponent contained in a positive sector $\Sigma_{\theta, c>0}$. Then the heat function associated to $S$ has an asymptotic expansion of order $\delta^{\prime} \geq-\infty$ with respect to the asymptotic sequence (see for example [9] for the definition) $\left\{t^{-\delta} \log ^{l} t\right\}_{\delta}$ as $t \rightarrow 0^{+}$if and only if the logarithmic $\Gamma$-function associated to $S$ has an asymptotic expansion of order $\alpha^{\prime} \geq-\infty$ with respect to the asymptotic sequence $\left\{(-\lambda)^{\alpha} \log ^{k}(-\lambda)\right\}_{\alpha}$ as $\lambda \rightarrow \infty$ uniformly in $\lambda$ for $\lambda \in B$, where $B$ is any unbounded region contained in the complement of $\Sigma_{\theta, c}$ (and $\delta^{\prime}=-\infty$ if and only if $\left.\alpha^{\prime}=-\infty\right)$. The order $\delta^{\prime}$ and the coefficients in the expansion of the heat function can be obtained from $\alpha^{\prime}$ and the coefficients in the expansion of the logarithmic Gamma function, but not vice versa.

Even if many useful results hold for a generic sequence of $S$-type, we consider here only sequences of a particular subclass as defined below (see also [1], Section 9).

Definition 2.6. A sequence of spectral type $S$ is called regular if the coefficients $a_{\alpha, k}$ in the expansion of $\log \Gamma(-\lambda, S)$ vanish for all $k \neq 0,1$.

Remark 2.7. Let $S$ be a regular sequence of spectral type with o $(S)=\alpha_{N}$. Then

$$
\log \Gamma(-\lambda, S)=\sum_{j=0}^{N} a_{\alpha_{j}, 0}(-\lambda)^{\alpha_{j}}+\sum_{j=0}^{N} a_{\alpha_{j}, 1}(-\lambda)^{\alpha_{j}} \log (-\lambda)+O\left((-\lambda)^{\alpha_{N}}\right),
$$

for large $\lambda$ in $A D_{\theta, a}(S)$ and where $\alpha_{0}>\alpha_{1}>\cdots>\alpha_{N}=\mathrm{o}(S)$.

Remark 2.8. If $S$ is a regular sequence of $S$-type, then $\alpha_{0} \leq \mathrm{e}(S)$, and $\alpha_{0}<\mathrm{g}(S)+1$.

We now give the main results on regular sequences of $S$-type in this context. The first result follows from equation (6) and properties of special functions.

Proposition 2.9 ([14]). Let $S$ be a regular sequence of spectral type with finite exponent and order $\alpha_{N}$. Then, the associated heat function has the following asymptotic expansion for $t \rightarrow 0^{+}$:

$$
f(t, S)-1=\sum_{j=0}^{N} \sum_{k=0}^{1} c_{\alpha_{j}, k} t^{-\alpha_{j}} \log ^{k} t+o\left(t^{-\alpha_{N}}\right)
$$


where $c_{\alpha_{j}, 0}=\frac{1}{\Gamma\left(-\alpha_{j}\right)}\left(a_{\alpha_{j}, 0}+\psi\left(-\alpha_{j}\right) a_{\alpha_{j}, 1}\right), c_{\alpha_{j}, 1}=-\frac{a_{\alpha_{j}, 1}}{\Gamma\left(-\alpha_{j}\right)}$, and $\psi$ is the Digamma function.

Note that the equations among the coefficients given in Proposition 2.9 are invertible provided $\alpha_{j} \notin \mathbb{N}$; moreover, $c_{\alpha_{j}, 1}=0$ whenever $\alpha_{j} \in \mathbb{N}$. Note also that the formulas in Proposition 2.9 for the coefficients only hold for regular sequences of spectral type. For a generic $S$-sequence, it is not true for example that the coefficients $c_{\alpha_{j}, 1}$ are null for $\alpha_{j}$ a natural number.

Next, we give formulas for the zeta invariants of a regular sequence. This result corresponds to the classical result for the functional zeta function of a differential operator. See also Theorems 3.4 and 3.6 of [5] for a further generalization to a larger class of sequences.

Proposition 2.10. Let $S$ be a regular sequence of spectral type of order $\alpha_{N}$. Then, the associated zeta function is regular in the complex half-plane $\operatorname{Re}(s)>\alpha_{N}-\epsilon$ (positive small $\epsilon$ ), up to a finite set of poles. The poles in the half-plane $\operatorname{Re}(s)>$ $\alpha_{N}-\epsilon$ are at most $N+1$, are located at $s=\alpha_{N}, \alpha_{N-1}, \ldots, \alpha_{0} \leq \mathrm{e}(S)$, and are of order at most 2 with residues

$$
\begin{aligned}
& \operatorname{Res}_{s=\alpha_{j}} \zeta(s, S)=-\frac{c_{\alpha_{j}, 1}}{\Gamma\left(\alpha_{j}\right)} \quad\left(=0, \text { if } \alpha_{j} \in \mathbb{Z}\right), \\
& \operatorname{Res}_{1} \zeta(s, S)= \begin{cases}\frac{c_{\alpha_{j}, 0}}{\Gamma\left(\alpha_{j}\right)}+c_{\alpha_{j}, 1} \frac{\psi\left(\alpha_{j}\right)}{\Gamma\left(\alpha_{j}\right)}, & \alpha_{j} \neq 0,-1,-2, \ldots,-\left[\left|\alpha_{N}\right|\right], \\
(-1)^{-\alpha_{j}+1}\left(-\alpha_{j}\right) ! c_{\alpha_{j}, 1}, & \alpha_{j}=-1,-2, \ldots,-\left[\left|\alpha_{N}\right|\right],\end{cases} \\
& \operatorname{Res}_{s=\alpha_{j}} \zeta(s, S)=(-1)^{\alpha_{j}}\left(-\alpha_{j}\right) ! c_{\alpha_{j}, 0}, \quad \alpha_{j}=-1,-2, \ldots,-\left[\left|\alpha_{N}\right|\right] ;
\end{aligned}
$$

in particular, if $\alpha_{N} \leq 0$, then $s=0$ is a regular point with $\zeta(0, S)=c_{0,0}$.

It is important to observe that all the formulas given in Proposition 2.10 can be written using exclusively the coefficients $a_{\alpha_{j}, k}$ appearing in the asymptotic expansion of the $\Gamma$-function. This follows from Proposition 2.9 or by direct computations (see also Proposition 2.14 below).

Theorem 2.11 ([14]). If $S$ is a regular sequence of spectral type of order $\alpha_{N} \leq 0$, then the associated zeta function is regular at $s=0$, and near $s=0$,

$$
\zeta(s, S)=-a_{0,1}-a_{0,0} s+O\left(s^{2}\right) .
$$

We call a regular sequence of spectral type simply regular if the unique logarithmic terms appearing in the expansion of $\log \Gamma(-\lambda, S)$ are of the form $(-\lambda)^{k} \log (-\lambda)$, with integer $k$, and totally regular if also $k \geq 0$. It is clear that the zeta function of a simply regular sequence of order $\alpha_{N}$ has at most simple poles for $\operatorname{Re}(s) \geq \alpha_{N}$. It is also easy to see that, while there can be logarithmic terms in the expansion of the heat function of a simply regular sequence, namely of the type $t^{k} \log t$, with negative integer $k$, we have $c_{\alpha_{j}, 1}=0$ for all $\alpha_{j}$ for a totally regular sequence.

Remark 2.12. When $S$ is a totally regular sequence of spectral type with order $\alpha_{N}$ and genus $\mathrm{g}$, the asymptotic expansion of the associated spectral functions reads:

$$
\begin{aligned}
\log \Gamma(-\lambda, S) & =\sum_{j=0}^{\mathrm{g}} a_{j, 1}(-\lambda)^{j} \log (-\lambda)+\sum_{j=0}^{N} a_{\alpha_{j}, 0}(-\lambda)^{\alpha_{j}}+o\left((-\lambda)^{\alpha_{N}}\right), \\
f(t, S)-1 & =\sum_{j=0}^{N} c_{\alpha_{j}, 0} t^{-\alpha_{j}}+o\left(t^{-\alpha_{N}}\right) .
\end{aligned}
$$


The main results concerning totally regular sequences are the following ones. First, we have formulas that express the coefficients $a_{\alpha_{j}, k}$ as functions of the coefficients $c_{\alpha_{j}, k}$ and some particular values of the zeta function (Proposition 2.13). Second, using the coefficients $a_{\alpha_{j}, k}$, we obtain additional information on the values of the residues of the associated zeta function (Proposition 2.14).

Proposition 2.13. Let $S$ be a totally regular sequence of spectral type with order $\alpha_{N}$ and genus $\mathrm{g}$. Then (recall $\left.\alpha_{j} \leq \mathrm{g}\right)$ :

$$
\begin{aligned}
& a_{\alpha_{j}, 0}= \begin{cases}\Gamma\left(-\alpha_{j}\right) c_{\alpha_{j}, 0} & \left(\alpha_{j} \notin \mathbb{Z}\right) \text { or }\left(\alpha_{j} \in \mathbb{Z} \text { and } \alpha_{j} \leq-1\right), \\
\frac{(-1)^{\alpha_{j}}}{\alpha_{j}}\left(\frac{1}{\alpha_{j} !} c_{\alpha_{j}, 0}-\operatorname{Res}_{s=\alpha_{j}} \zeta(s, S)\right) & \alpha_{j} \in \mathbb{Z} \text { and } 0<\alpha_{j} \leq \mathrm{g}, \\
-\operatorname{Res}_{s=0} \zeta^{\prime}(s, S) & \alpha_{j}=0,\end{cases} \\
& a_{\alpha_{j}, 1}= \begin{cases}0 & \left(\alpha_{j} \notin \mathbb{Z}\right) \text { or }\left(\alpha_{j} \in \mathbb{Z} \text { and } \alpha_{j} \leq-1\right), \\
\frac{(-1)^{\alpha_{j}+1}}{\alpha_{j} !} c_{\alpha_{j}, 0} & \alpha_{j} \in \mathbb{Z} \text { and } 0<\alpha_{j} \leq \mathrm{g}, \\
-\operatorname{Res}_{s=0} \zeta(s, S)=-c_{0,0} & \alpha_{j}=0 .\end{cases}
\end{aligned}
$$

Proof. We adapt here a technique introduced in [12. Integrate g times with respect to $t$ in equation (6). We obtain

$$
\zeta(s, S)=\frac{(-1)^{\mathrm{g}} s}{\Gamma(s-\mathrm{g})} \int_{0}^{\infty} t^{s-\mathrm{g}-1} \frac{1}{2 \pi i} \int_{\Lambda_{\theta, c}} \frac{\mathrm{e}^{-\lambda t}}{(-\lambda)^{\mathrm{g}+1}} \log \Gamma(-\lambda, S) d \lambda d t .
$$

Next, split the $t$-integral at $t=1$. The $t$-integral $\int_{1}^{\infty}$ is a regular function of $s$; in the $t$-integral $\int_{0}^{1}$, we change the contour to $\Lambda_{\theta,-c}-C_{c}$, where $C_{c}$ is a circle with center at the origin and radius $c$, and the $\lambda$ integration along $C_{c}$ vanishes, since by definition, $\operatorname{Res}_{\lambda=0} \frac{\mathrm{e}^{-\lambda t}}{(-\lambda)^{\mathrm{g}}} \log \Gamma(-\lambda, S)=0$ (in fact $\log \Gamma(-\lambda, S)$ has a zero of order $\mathrm{g}+1$ at $\lambda=0)$. Thus,

$$
\zeta(s, S)=\frac{(-1)^{\mathrm{g}} s}{\Gamma(s-\mathrm{g})}\left(\int_{0}^{1} t^{s-1} \frac{1}{2 \pi i} \int_{\Lambda_{\theta,-c}} \frac{\mathrm{e}^{-\lambda}}{(-\lambda)^{\mathrm{g}+1}} \log \Gamma(-\lambda / t, S) d \lambda d t+r(s)\right),
$$

where $r(s)$ is regular for all $s\left(\operatorname{Re}(s)>\alpha_{N}-\epsilon\right)$. We use the expansion of the $\Gamma$-function and equations (13) and (14) in the appendix in order to obtain

$$
\begin{array}{r}
\zeta(s, S)=\frac{(-1)^{\mathrm{g}+1} s}{\Gamma(s-\mathrm{g})}\left(\sum_{j=0}^{N} \frac{1}{\Gamma\left(\mathrm{g}+1-\alpha_{j}\right)} \frac{a_{\alpha_{j}, 0}}{s-\alpha_{j}}+\sum_{j=0}^{N} \frac{\psi\left(\mathrm{g}+1-\alpha_{j}\right)}{\Gamma\left(\mathrm{g}+1-\alpha_{j}\right)} \frac{a_{\alpha_{j}, 1}}{s-\alpha_{j}}\right. \\
\left.+\sum_{j=0}^{N} \frac{1}{\Gamma\left(\mathrm{g}+1-\alpha_{j}\right)} \frac{a_{\alpha_{j}, 1}}{\left(s-\alpha_{j}\right)^{2}}+r(s)\right) .
\end{array}
$$

By direct inspection of the above equation, since the zeta function cannot have double poles and $S$ is totally regular, it follows that $a_{\alpha_{j}, 1}=0$, whenever $\alpha_{j}$ is not an integer, or $\alpha_{j}$ is an integer and $\alpha_{j} \leq-1$ or $\alpha_{j} \geq \mathrm{g}+1$. Now, if $\alpha_{j}$ is not an integer or it is an integer and $\alpha_{j} \leq-1$ or $\alpha_{j} \geq \mathrm{g}+1$, then the zeta function has a pole at $s=\alpha_{j}$ and

$$
\operatorname{Res}_{s=\alpha_{j}} \zeta(s, S)=\frac{(-1)^{\mathrm{g}+1} \alpha_{j}}{\Gamma\left(\alpha_{j}-\mathrm{g}\right)} \frac{a_{\alpha_{j}, 0}}{\Gamma\left(\mathrm{g}+1-\alpha_{j}\right)}=-\alpha_{j} \frac{\sin \alpha_{j} \pi}{\pi} a_{\alpha_{j}, 0} .
$$


Therefore, since by Propositions 2.9 and 2.10

$$
\operatorname{Res}_{s=\alpha_{j}} \zeta(s, S)=\frac{c_{\alpha_{j}, 0}}{\Gamma\left(\alpha_{j}\right)},
$$

we obtain that $a_{\alpha_{j}, 0}=\Gamma\left(-\alpha_{j}\right) c_{\alpha_{j}, 0}$. If $\alpha_{j}$ is an integer and $0 \leq \alpha_{j} \leq \mathrm{g}$, using the expansion for $s$ near $\alpha_{j}=k$ of $\frac{s(s-1) \ldots(s-\mathrm{g})}{\Gamma(s)}=\frac{s}{\Gamma(s-\mathrm{g})}$ in equation (8), we obtain after some calculations,

$$
\zeta(s, S)=(-1)^{k+1}(s-k+k)\left(\frac{a_{k, 1}}{s-k}+a_{k, 0}+O(s-k)\right) .
$$

We distinguish two cases. First, if $k=0$, then near $s=0$,

$$
\zeta(s, S)=-a_{0,1}-a_{0,0} s+O\left(s^{2}\right),
$$

as in Theorem 2.11 Second, if $0<k \leq \mathrm{g}$, then near $s=k$,

$$
\zeta(s, S)=(-1)^{k+1} k \frac{a_{k, 1}}{s-k}+(-1)^{k+1} k a_{k, 0}+(-1)^{k+1} a_{k, 1}+O(s-k),
$$

and using Proposition 2.10, this completes the proof.

Proposition 2.14. Let $S$ be a totally regular sequence of spectral type with order $\alpha_{N}$ and genus $\mathrm{g}$. Then, the associated zeta function is regular in the complex halfplane $\operatorname{Re}(s)>\alpha_{N}-\epsilon$ up to a finite set of at most $N+1$ simple poles located at $s=\alpha_{N}, \alpha_{N-1}, \ldots, \alpha_{0}$ :

$$
\begin{aligned}
& \operatorname{Res}_{s=\alpha_{j}} \zeta(s, S)=\frac{a_{\alpha_{j}, 0}}{\Gamma\left(\alpha_{j}\right) \Gamma\left(-\alpha_{j}\right)}, \quad \alpha_{j} \neq-\left[\left|\alpha_{N}\right|\right], \ldots, \mathrm{g}, \\
& \operatorname{Res}_{s=\alpha_{j}} \zeta(s, S)= \begin{cases}(-1)^{\alpha_{j}+1} \alpha_{j} a_{\alpha_{j}, 1}, & \alpha_{j}=1, \ldots, \mathrm{g}, \\
0, & \alpha_{j}=-\left[\left|\alpha_{N}\right|\right], \ldots, 0,\end{cases} \\
& \operatorname{Res}_{s=\alpha_{j}} \zeta(s, S)= \begin{cases}(-1)^{\alpha_{j}+1} \alpha_{j} a_{\alpha_{j}, 0}, & \alpha_{j}=-\left[\left|\alpha_{N}\right|\right], \ldots,-1, \\
(-1)^{\alpha_{j}+1}\left(\alpha_{j} a_{\alpha_{j}, 0}+a_{\alpha_{j}, 1}\right), & \alpha_{j}=1, \ldots, \mathrm{g}, \\
-a_{\alpha_{j}, 1}, & \alpha_{j}=0 .\end{cases}
\end{aligned}
$$

We conclude this section with some properties of sums and multiplication by a scalar of sequences of spectral type. All the proofs are straightforward, up to 2.17 where we can use the Young inequality. Let $S=\left\{\lambda_{n}\right\}_{n \in \mathbb{N}_{0}}$ be a sequence and $y$ a positive number. Denote by $y S$ the sequence $y S=\left\{y \lambda_{n}\right\}_{n \in \mathbb{N}_{0}}$. Let $n=\left(n_{1}, \ldots, n_{I}\right)$ be an integer vector. If $S_{i}=\left\{\lambda_{(i), n_{i}}\right\}_{n_{i} \in \mathbb{N}_{0}}, i=1, \ldots, I$, is a finite set of sequences, we use the notation $\sum_{i=1}^{I} S_{(i)}$ for the sum of the sequences, namely the sequence $\left\{\lambda_{n}=\sum_{i=1}^{I} \lambda_{(i), n_{1}}\right\}_{n \in\left(\mathbb{N}_{0}\right)^{I}}$.

Lemma 2.15. For any positive real $y, S=\left\{\lambda_{n}\right\}_{n \in \mathbb{N}_{0}}$ is a sequence with finite exponent $\mathrm{e}$, genus $\mathrm{g}$ and spectral sector $\Sigma_{\theta, c}$ if and only if $y S$ is a sequence with finite exponent $\mathrm{e}$, genus $\mathrm{g}$, and spectral sector $\Sigma_{\theta, y c} . S$ is of spectral type of order $\alpha_{N}$ if and only if $y S$ is of spectral type of order $\alpha_{N}$; $S$ is regular, simply or totally regular if and only if $y S$ is regular, simply or totally regular respectively.

Lemma 2.16. If $S_{(i)}=\left\{\lambda_{(i), n_{i}}\right\}_{n_{i} \in \mathbb{N}_{0}}, i=1, \ldots, I$, is a finite family of sequences, then

$$
f\left(t, \sum_{i=1}^{I} S_{(i)}\right)=1+\prod_{i=1}^{I}\left(f\left(t, S_{(i)}\right)-1\right) .
$$


Lemma 2.17. If $S_{(i)}=\left\{\lambda_{(i), n_{i}}\right\}_{n_{i} \in \mathbb{N}_{0}}, i=1, \ldots, I$, is a finite family of sequences of spectral type of finite exponents $\mathrm{e}_{(i)}$, genus $\mathrm{g}_{(i)}$, asymptotic domains $A D_{\theta_{(i)}, c_{(i)}}$, and orders $\alpha_{(i), N_{(i)}}$, then the sum sequence $S=\sum_{i=1}^{I} S_{(i)}$ is a sequence of spectral type with exponent $\mathrm{e}_{(0)}=\sum_{i=1}^{I} \mathrm{e}_{(i)}$, asymptotic domain $A D_{\theta, c}$, where $\theta=\max \left(\theta_{(i)}\right)$ and $c=\min \left(c_{(i)}\right)$, and order $\alpha_{N}=\max \left(\alpha_{(i), N(i)}\right)$.

Lemma 2.18. If $S_{(i)}=\left\{\lambda_{(i), n_{i}}\right\}_{n_{i} \in \mathbb{N}_{0}}, i=1, \ldots, I$, is a finite family of simply/totally regular sequences of spectral type, then the sum sequence $S=\sum_{i=1}^{I} S_{(i)}$ is a simply/totally regular sequence of spectral type.

\section{Spectral decomposition}

The aim of this section is to obtain the main zeta invariants of double sequences. It is clear that, using multi-indices, the results extend to multiple sequences. Our strategy to deal with double sequences is to define a class of double sequences that can be decomposed as sums of simple sequences relative to some fixed simple sequence in a suitable way. The double sequences of this class are said to be spectrally decomposable and are defined in Definition 3.1. The idea of this type of decomposition was suggested by the results of Brüning and Seeley (in particular, see [1]) and has already been applied with success in a number of cases ([13], [15]). For the zeta function associated to spectrally decomposable double sequences, we obtain conditions for regularity at $s=0$ and a formula that gives the values of $\zeta(0)$ and $\zeta^{\prime}(0)$ (Theorem 3.9).

Let $S=\left\{\lambda_{n, k}\right\}_{n, k=1}^{\infty}$ be a double sequence of nonvanishing complex numbers with unique accumulation point the infinity, finite exponent $s_{0}=\mathrm{e}(S)$ and genus $p_{0}=\mathrm{g}(S)$. Assume if necessary that the elements of $S$ are ordered as $0<\left|\lambda_{1,1}\right| \leq$ $\left|\lambda_{1,2}\right| \leq\left|\lambda_{2,1}\right| \leq \ldots$ We use the notation $S_{n}\left(S_{k}\right)$ to denote the simple sequence with fixed $n(k)$. We call the exponents of $S_{n}$ and $S_{k}$ the relative exponents of $S$, and we use the notation $\left(s_{0}=\mathrm{e}(S), s_{1}=\mathrm{e}\left(S_{k}\right), s_{2}=\mathrm{e}\left(S_{n}\right)\right)$. We define the relative genus accordingly.

Definition 3.1. Let $S=\left\{\lambda_{n, k}\right\}_{n, k=1}^{\infty}$ be a double sequence with finite exponents $\left(s_{0}, s_{1}, s_{2}\right)$, genus $\left(p_{0}, p_{1}, p_{2}\right)$, and positive spectral sector $\Sigma_{\theta_{0}, c_{0}}$. Let $U=\left\{u_{n}\right\}_{n=1}^{\infty}$ be a totally regular sequence of spectral type of order $u^{\prime} \leq 0$ with exponent $r_{0}$, genus $q$, and domain $A D_{\phi, d}$. We say that $S$ is spectrally decomposable over $U$ with power $\kappa$, length $\ell$ and asymptotic domain $A D_{\theta, c}$, with $c=\min \left(c_{0}, d, c^{\prime}\right), \theta=\max \left(\theta_{0}, \phi, \theta^{\prime}\right)$, if there exist positive real numbers $\kappa, \ell$ (integer), $c^{\prime}$, and $\theta^{\prime}$, with $0<\theta^{\prime}<\pi$, such that:

(1) the sequence $\widetilde{S}_{n}=u_{n}^{-\kappa} S_{n}=\left\{\frac{\lambda_{n, k}}{u_{n}^{\kappa}}\right\}_{k=1}^{\infty}$ has spectral sector $\Sigma_{\theta^{\prime}, c^{\prime}}$ and is a totally regular sequence of spectral type of order $\leq 0$ for each $n$;

(2) the logarithmic $\Gamma$-function associated to $\widetilde{S}_{n}$ has an asymptotic expansion for large $n$ uniformly in $\lambda$ for $\lambda$ in $A D_{\theta, c}$ of the following form:

$\log \Gamma\left(-\lambda, u_{n}^{-\kappa} S_{n}\right)=\sum_{h=0}^{\ell} \phi_{\sigma_{h}}(\lambda) u_{n}^{-\sigma_{h}}+\sum_{l=0}^{L} P_{\rho_{l}}(\lambda) u_{n}^{-\rho_{l}} \log u_{n}+o\left(u_{n}^{-r_{0}}\right)$,

where $\sigma_{h}$ and $\rho_{l}$ are real numbers with $\sigma_{0}<\cdots<\sigma_{\ell}, \rho_{0}<\cdots<\rho_{L}$, the $P_{\rho_{l}}(\lambda)$ are polynomials in $\lambda$ satisfying the condition $P_{\rho_{l}}(0)=0, \ell$ and $L$ are the larger integers such that $\sigma_{\ell} \leq r_{0}$ and $\rho_{L} \leq r_{0}$, and $\sigma_{0}$ and $\rho_{0}$ satisfy the condition $u^{\prime}<\min \left(\sigma_{0}, \rho_{0}-1\right)$. 
We point out that the condition $P_{\rho_{l}}(0)=0$ is introduced in Definition 3.1 expressly in order to avoid the dependence of the final result on the logarithmic terms. More precisely, without this condition, the function $A_{0,0}(s)$, introduced in Lemma 3.5. would depend on the values of the coefficients of the polynomials $P_{\rho_{l}}(\lambda)$. As a consequence, also the coefficients of the expansion of the zeta function at zero given in Theorem 3.9 would depend on the coefficients of the $P_{\rho_{l}}(\lambda)$.

The proof of the following lemma is essentially based on standard properties of double limits.

Lemma 3.2. The functions $\phi_{\sigma_{h}}(\lambda)$ appearing in Definition 3.1 have an asymptotic expansion of order $\leq 0$ for large $\lambda$ in $A D_{\theta, c}$ with respect to the asymptotic sequence $\left\{(-\lambda)^{\alpha} \log ^{k}(-\lambda)\right\}_{\alpha}, k=0,1$, where the unique logarithmic terms are of the form $(-\lambda)^{m} \log (-\lambda)$, with integer $m$ such that $m \leq p_{2}$. The maximum order of the polynomials $P_{\rho_{l}}(\lambda)$ is less than or equal to $p_{2}$.

When $S$ is a double sequence, we consider the general case where we do not know an explicit expansion for $\log \Gamma(-\lambda, S)$, and hence we cannot apply Theorem 2.11. On the other side we suppose that we do know such expansions for the sequences $\widetilde{S}_{n}$, and our aim is to use this information to obtain the result for $S$. We could write $\log \Gamma(-\lambda, S)=\sum_{n=1}^{\infty} \log \Gamma\left(-\lambda, S_{n}\right)$ and use this decomposition in the formula (6) for $\zeta(s, S)$. Then, we could proceed as in the proof of Proposition 2.13 using the known information on the expansion of $S_{n}$, and eventually try to sum on $n$. This procedure does not work. The series in $n$ may not converge. To overcome this difficulty, the idea is to insert an $s$ in the general term. In other words, we decompose the zeta function as $\zeta(s, S)=\sum_{n=1}^{\infty} u_{n}^{-\kappa s} \zeta\left(s, u_{n}^{-\kappa} S_{n}\right)$ (see Lemma 3.4 below). The power $\kappa$ is necessary to guarantee the existence of an asymptotic expansion of $\log \Gamma\left(-\lambda, \widetilde{S}_{n}\right)$ for large $n$.

Lemma 3.3. Let $S$ be spectrally decomposable over $U$ as in Definition 3.1. Then, there exist constants $N, \alpha_{N}<\cdots<\alpha_{0}, a_{\alpha_{j}, k, n}$ and $b_{\sigma_{h}, \alpha_{j}, k}$, with $\alpha_{N} \leq 0$, such that

$$
\log \Gamma\left(-\lambda, \widetilde{S}_{n}\right)=\sum_{j=0}^{N} \sum_{k=0}^{1} a_{\alpha_{j}, k, n}(-\lambda)^{\alpha_{j}} \log ^{k}(-\lambda)+o\left((-\lambda)^{\alpha_{N}}\right),
$$

for all $n$, and

$$
\phi_{\sigma_{h}}(\lambda)=\sum_{j=0}^{N} \sum_{k=0}^{1} b_{\sigma_{h}, \alpha_{j}, k}(-\lambda)^{\alpha_{j}} \log ^{k}(-\lambda)+o\left((-\lambda)^{\alpha_{N}}\right),
$$

for all $\sigma_{h}$, for large $\lambda$ uniformly in $A D_{\theta, c}$, and where $a_{\alpha_{j}, 1, n}=b_{\sigma_{h}, \alpha_{j}, 1}=0$ for all $\alpha_{j} \neq 0,1, \ldots, p_{2}$.

Proof. Since $\widetilde{S}_{n}=u_{n}^{-\kappa} S_{n}$ is of spectral type for each $n$, we have that the sequences of powers $\alpha_{j}$ appearing in the asymptotic expansion of the $\Gamma$-functions are all upper bounded by $s_{1}$ (by Proposition 2.10). Hence, they can all only accumulate at $-\infty$, and hence we can collect them in a unique sequence $\left\{\alpha_{j}\right\}_{j=0}^{N}$, starting at the larger nonvanishing one and where $\alpha_{N}$ is the smaller of the order of the $\widetilde{S}_{n}$. Notice also that since the expansions of the $\log \Gamma\left(-\lambda, S_{n}\right)$ are all of order $\leq 0$, it follows that $\alpha_{N} \leq 0$. For the domain, if the conditions in Definition 3.1 are satisfied, all the $\widetilde{S}_{n}$ are contained in the positive spectral sector $\Sigma_{\theta, c}$. The same argument works for the $\phi_{\sigma_{h}}$, and again we can reset $\alpha_{N}$ to be the smaller of the orders and it will 
always be negative or null. The condition on the coefficients follows from the point (1) (the fact that $\widetilde{S}_{n}$ is totally regular) of Definition 3.1 (see also Remark 2.12).

Lemma 3.4. Let $S$ be spectrally decomposable over $U$ as in Definition 3.1. Then, we have the following contour integral representation for the associated zeta function:

$$
\zeta(s, S)=\frac{s}{\Gamma(s)} \int_{0}^{\infty} t^{s-1} \frac{1}{2 \pi i} \int_{\Lambda_{\theta, c}} \frac{\mathrm{e}^{-\lambda t}}{-\lambda} \mathcal{T}(s, \lambda, S, U) d \lambda,
$$

where

$$
\mathcal{T}(s, \lambda, S, U)=\sum_{n=1}^{\infty} u_{n}^{-\kappa s} \log \Gamma\left(-\lambda, u_{n}^{-\kappa} S_{n}\right) .
$$

Proof. By uniform convergence of the series, for $\operatorname{Re}(s)>s_{0}$, we can write

$$
\zeta(s, S)=\sum_{n=1}^{\infty} u_{n}^{-\kappa s} \zeta\left(s, u_{n}^{-\kappa} S_{n}\right)
$$

where

$$
\zeta\left(s, u_{n}^{-\kappa s} S_{n}\right)=u_{n}^{-\kappa s} \sum_{k=1}^{\infty} \lambda_{n, k}^{-s}
$$

is well defined by the series since $s_{0} \geq s_{2}$. Now, applying equation (7) of Lemma2.3 to $\zeta\left(s, u_{n}^{-\kappa s} S_{n}\right)$, when $\operatorname{Re}(s)>s_{0} \geq s_{1}, s_{2}$, thanks to uniform convergence of the integral, we obtain the thesis.

Lemma 3.5. Let $S$ be spectrally decomposable over $U$ as in Definition 3.1. Then, the function $\mathcal{T}(s, \lambda, S)$ can be extended analytically to the half-plane $\operatorname{Re}(s)>-\epsilon$ (positive small $\epsilon$ ) by the following formula for all $\lambda \in A D_{\theta, c}$ :

$\mathcal{T}(s, \lambda, S, U)=\mathcal{P}(s, \lambda, S, U)+\sum_{h=0}^{\ell} \phi_{\sigma_{h}}(\lambda) \zeta\left(\kappa s+\sigma_{h}, U\right)-\sum_{l=0}^{L} P_{\rho_{l}}(\lambda) \zeta^{\prime}\left(\kappa s+\rho_{l}, U\right)$.

Moreover, the function $\mathcal{P}(s, \lambda, S, U)$ is regular in $s$ for $\operatorname{Re}(s)>-\epsilon$ and has the following expansion for large $\lambda$ uniformly in $A D_{\theta, c}$ :

$$
\mathcal{P}(s, \lambda, S, U)=\sum_{j=0}^{N} A_{\alpha_{j}, 0}(s)(-\lambda)^{\alpha_{j}}+\sum_{j=0}^{p_{2}} A_{j, 1}(s)(-\lambda)^{j} \log (-\lambda)+o\left((-\lambda)^{\alpha_{N}}\right),
$$

where $N$ is defined in Lemma 3.3 and the coefficients

$$
\begin{aligned}
A_{\alpha_{j}, 0}(s) & =\sum_{n=1}^{\infty}\left(a_{\alpha_{j}, 0, n}-\sum_{h=0}^{\ell} b_{\sigma_{h}, \alpha_{j}, 0} u_{n}^{-\sigma_{h}}\right) u_{n}^{-\kappa s}, \alpha_{j} \neq 0,1, \ldots, p_{2}, \\
A_{0,0}(s) & =\sum_{n=1}^{\infty}\left(a_{0,0, n}-\sum_{h=0}^{\ell} b_{\sigma_{h}, 0,0} u_{n}^{-\sigma_{h}}\right) u_{n}^{-\kappa s}, \\
A_{j, 0}(s) & =\sum_{n=1}^{\infty}\left(a_{j, 0, n}-\sum_{h=0}^{\ell} b_{\sigma_{h}, j, 0} u_{n}^{-\sigma_{h}}-\sum_{l=0}^{L} p_{\rho_{l}, j} u_{n}^{-\rho_{l}} \log u_{n}\right) u_{n}^{-\kappa s}, 1 \leq j \leq p_{2}, \\
A_{j, 1}(s) & =\sum_{n=1}^{\infty}\left(a_{j, 1, n}-\sum_{h=0}^{\ell} b_{\sigma_{h}, j, 1} u_{n}^{-\sigma_{h}}\right) u_{n}^{-\kappa s}, 0 \leq j \leq p_{2},
\end{aligned}
$$

are regular functions of $s$ for $\operatorname{Re}(s)>-\epsilon$. 
Noting that $\mathcal{T}$ and $\mathcal{P}$ are regular does not mean that $\zeta(s, S)$ is regular, just that we need not bother about the dependence on $s$ coming from $\mathcal{T}$.

Proof. We would like to expand $\mathcal{T}$ for large $\lambda$ in order to proceed as in the proof of Proposition 2.13. We could use the expansion of $\log \Gamma\left(-\lambda, u_{n}^{-\kappa} S_{n}\right)$ for large $\lambda$ to get that of $\mathcal{T}$. Unfortunately, this does not work for the following reason. When we expand $\mathcal{T}$ and we perform the integrals in $\lambda$ and $t$ for each term, the resulting functions have poles in $s$ at $s=0$ and the sum over $n$ also gives a pole in the same point; thus we get some terms with a double pole, and hence we cannot use the formula (6) to compute the derivative in $s$ at $s=0$. What we can do is to split into two terms, each one having only simple poles. We do this as follows. Since $S$ is spectrally decomposable over $U$ with power $\kappa$, we have the expansion (observe that $\left.\log \Gamma\left(-\lambda, \widetilde{S}_{n}\right)=\log \Gamma\left(-\lambda u_{n}^{\kappa}, S_{n}\right)\right)$

$$
\log \Gamma\left(-\lambda, \widetilde{S}_{n}\right)=\sum_{h=0}^{\ell} \phi_{\sigma_{h}}(\lambda) u_{n}^{-\sigma_{h}}+\sum_{l=0}^{L} P_{\rho_{l}}(\lambda) u_{n}^{-\rho_{l}} \log u_{n}+o\left(u_{n}^{-K}\right) .
$$

This allows us to split $\mathcal{T}$ into two terms:

$$
\begin{aligned}
\mathcal{T}(s, \lambda, S, U) & =\mathcal{P}(s, \lambda, S, U)+\sum_{h=0}^{\ell} \sum_{n=1}^{\infty} \phi_{\sigma_{h}}(\lambda) u_{n}^{-\kappa s-\sigma_{h}}+\sum_{l=0}^{L} P_{\rho_{l}}(\lambda) u_{n}^{-\kappa s-\rho_{l}} \log u_{n} \\
& =\mathcal{P}(s, \lambda, S, U)+\sum_{h=0}^{\ell} \phi_{\sigma_{h}}(\lambda) \zeta\left(\kappa s+\sigma_{h}, U\right)-\sum_{l=0}^{L} P_{\rho_{l}}(\lambda) \zeta^{\prime}\left(\kappa s+\rho_{l}, U\right)
\end{aligned}
$$

where

$\mathcal{P}(s, \lambda, S, U)=\sum_{n=1}^{\infty} u_{n}^{-\kappa s}\left(\log \Gamma\left(-\lambda u_{n}^{\kappa}, S_{n}\right)-\sum_{h=0}^{\ell} \phi_{\sigma_{h}}(\lambda) u_{n}^{-\sigma_{h}}-\sum_{l=0}^{L} P_{\rho_{l}}(\lambda) u_{n}^{-\rho_{l}} \log u_{n}\right)$.

Since, by Definition 3.1, the term in brackets in the above sum is $O\left(u_{n}^{-r_{0}-\epsilon}\right)$ uniformly in $\lambda$ for $\lambda \in A D_{\theta, c}$, it follows that

$$
\mathcal{P}(s, \lambda, S, U)<\sum_{n=1}^{\infty} u_{n}^{-\kappa s-r_{0}-\epsilon},
$$

and hence $\mathcal{P}$ is regular when $\operatorname{Re}\left(\kappa s+r_{0}+\epsilon\right) \geq r_{0}$, i.e. $\operatorname{Re}(s) \geq-\frac{\epsilon}{\kappa}$. As $\kappa>0, \mathcal{P}$ is regular at $s=0$, uniformly for $\lambda$ in $A D_{\theta, c}$. Thus we can get the expansion of $\mathcal{P}$ as follows. It is clear that the expansion for large $\lambda$ of $\log \Gamma\left(-\lambda, S_{n}\right)$ gives that of $\log \Gamma\left(-\lambda u_{n}^{\kappa}, S_{n}\right)$ ( $n$ fixed). Thus we can use the expansions given in Lemma 3.3. Substituting in the definition of $\mathcal{P}$ we have

$$
\begin{aligned}
\mathcal{P}(s, \lambda, S, U)=\sum_{n=1}^{\infty} u_{n}^{-\kappa s} \sum_{j=0}^{N} \sum_{k=0}^{1} & \left(a_{\alpha_{j}, k, n}(-\lambda)^{\alpha_{j}} \log ^{k}(-\lambda)-\sum_{l=0}^{L} P_{\rho_{l}}(\lambda) u_{n}^{-\rho_{l}} \log u_{n}\right. \\
& \left.-\sum_{h=0}^{\ell} b_{\sigma_{h}, \alpha_{j}, k} u_{n}^{-\sigma_{h}}(-\lambda)^{\alpha_{j}} \log ^{k}(-\lambda)\right)+o\left((-\lambda)^{\alpha_{N}}\right),
\end{aligned}
$$


and since the series defining $\mathcal{P}$ is uniformly convergent for $\operatorname{Re}(s)>s_{2}$,

$$
\begin{aligned}
& \mathcal{P}(s, \lambda, S, U)=\sum_{n=1}^{\infty} u_{n}^{-\kappa s} \sum_{j=0}^{N}\left(a_{\alpha_{j}, 0, n}(-\lambda)^{\alpha_{j}}-\sum_{h=0}^{\ell} b_{\sigma_{h}, \alpha_{j}, 0} u_{n}^{-\sigma_{h}}(-\lambda)^{\alpha_{j}}\right) \\
&+\sum_{n=1}^{\infty} u_{n}^{-\kappa s} \sum_{j=0}^{p_{2}}\left(a_{j, 1, n}(-\lambda)^{j} \log (-\lambda)-\sum_{l=0}^{L} P_{\rho_{l}}(\lambda) u_{n}^{-\rho_{l}} \log u_{n}\right. \\
&\left.\quad-\sum_{h=0}^{\ell} b_{\sigma_{h}, j, 1} u_{n}^{-\sigma_{h}}(-\lambda)^{j} \log (-\lambda)\right)+o\left((-\lambda)^{\alpha_{N}}\right) .
\end{aligned}
$$

Writing (recall $\left.P_{\rho_{l}}(0)=0\right) P_{\rho_{l}}(\lambda)=\sum_{m=1}^{G_{\rho_{l}}} p_{\rho_{l}, m}(-\lambda)^{m}$, we have

$$
\sum_{l=0}^{L} P_{\rho_{l}}(\lambda) \zeta^{\prime}\left(\kappa s+\rho_{l}, U\right)=\sum_{m=1}^{G}\left(\sum_{l=0}^{L} p_{\rho_{l}, m} \zeta^{\prime}\left(\kappa s+\rho_{l}, U\right)\right)(-\lambda)^{m}
$$

where $G=\max \left(G_{\rho_{l}}\right) \leq p_{2}$, and we set $p_{\rho_{l}, m}=0$ for $m>G_{\rho_{l}}$; then

$$
\begin{aligned}
\mathcal{P}(s, \lambda, S, U)= & \sum_{n=1}^{\infty} \sum_{j=0}^{N}\left(a_{\alpha_{j}, 0, n}-\sum_{h=0}^{\ell} b_{\sigma_{h}, \alpha_{j}, 0} u_{n}^{-\sigma_{h}}\right) u_{n}^{-\kappa s}(-\lambda)^{\alpha_{j}} \\
& -\sum_{n=1}^{\infty} \sum_{m=1}^{G}\left(\sum_{l=0}^{L} p_{\rho_{l}, m} u_{n}^{-\kappa s-\rho_{l}} \log u_{n}\right)(-\lambda)^{m} \\
& +\sum_{n=1}^{\infty} \sum_{j=0}^{p_{2}}\left(a_{j, 1, n}-\sum_{h=0}^{\ell} b_{\sigma_{h}, j, 1} u_{n}^{-\sigma_{h}}\right) u_{n}^{-\kappa s}(-\lambda)^{j} \log (-\lambda)+o\left((-\lambda)^{\alpha_{N}}\right) .
\end{aligned}
$$

This gives the coefficients stated in the thesis, and since $\mathcal{P}(s, \lambda, S, U)$ is regular at $s=0$, this also shows that they are regular at $s=0$.

Lemma 3.6. Let $S$ be spectrally decomposable over $U$ as in Definition 3.1. Then, near $s=0$, we have the following representation for the zeta function:

$$
\Gamma(s) \zeta(s, S)=\gamma A_{0,1}(s)-A_{0,0}(s)-\frac{A_{0,1}(s)}{s}+s \sum_{h=0}^{\ell} \Phi_{\sigma_{h}}(s) \zeta\left(\kappa s+\sigma_{h}, U\right)+s r(s),
$$

where $r(s)$ is regular near $s=0$ and

$$
\Phi_{\sigma_{h}}(s)=\int_{0}^{\infty} t^{s-1} \frac{1}{2 \pi i} \int_{\Lambda_{\theta, c}} \frac{\mathrm{e}^{-\lambda t}}{-\lambda} \phi_{\sigma_{h}}(\lambda) d \lambda d t .
$$

Proof. Let $\operatorname{Re}(s)>s_{0}$. By Lemma 3.4.

$$
\zeta(s, S)=\frac{s}{\Gamma(s)} \int_{0}^{\infty} t^{s-1} \frac{1}{2 \pi i} \int_{\Lambda_{\theta, c}} \frac{\mathrm{e}^{-\lambda t}}{-\lambda} \mathcal{T}(s, \lambda, S) d \lambda .
$$


Substituting the decomposition of $\mathcal{T}$ given by Lemma 3.5, we obtain

$$
\begin{gathered}
\zeta(s, S)=\zeta_{1}(s)+\zeta_{2}(s)+\zeta_{3}(s), \\
\zeta_{1}(s)=\frac{s}{\Gamma(s)} \int_{0}^{\infty} t^{s-1} \frac{1}{2 \pi i} \int_{\Lambda_{\theta, c}} \frac{\mathrm{e}^{-\lambda t}}{-\lambda} \mathcal{P}(s, \lambda, S, U) d \lambda d t \\
\zeta_{2}(s)=\frac{s}{\Gamma(s)} \sum_{h=0}^{H} \zeta\left(\kappa s+\sigma_{h}, U\right) \int_{0}^{\infty} t^{s-1} \frac{1}{2 \pi i} \int_{\Lambda_{\theta, c}} \frac{\mathrm{e}^{-\lambda t}}{-\lambda} \phi_{\sigma_{h}}(\lambda) d \lambda d t, \\
\zeta_{3}(s)=-\frac{s}{\Gamma(s)} \sum_{l=0}^{L} P_{\rho_{l}}(\lambda) \zeta^{\prime}\left(\kappa s+\rho_{l}, U\right) \int_{0}^{\infty} t^{s-1} \frac{1}{2 \pi i} \int_{\Lambda_{\theta, c}} \frac{\mathrm{e}^{-\lambda t}}{-\lambda} P_{\rho_{l}}(\lambda) d \lambda d t .
\end{gathered}
$$

Now, $\zeta_{2}(s)$ can immediately be written as stated in the thesis, while $\zeta_{3}(s)$ vanishes by direct calculation (using the integral given in the appendix). For $\zeta_{1}(s)$, we can use the expansion given in Lemma 3.5 for $\mathcal{P}$ as follows. First, split the integral $\left(\operatorname{Re}(s)>s_{0}\right)$

$$
\begin{aligned}
\zeta_{1}(s)= & \frac{s}{\Gamma(s)} \int_{0}^{1} t^{s-1} \frac{1}{2 \pi i} \int_{\Lambda_{\theta, c}} \frac{\mathrm{e}^{-\lambda t}}{-\lambda} \mathcal{P}(s, \lambda, S, U) d \lambda d t \\
& +\frac{s}{\Gamma(s)} \int_{1}^{\infty} t^{s-1} \frac{1}{2 \pi i} \int_{\Lambda_{\theta, c}} \frac{\mathrm{e}^{-\lambda t}}{-\lambda} \mathcal{P}(s, \lambda, S, U) d \lambda d t .
\end{aligned}
$$

For the second term, note that $\mathcal{P}$ is regular for $\operatorname{Re}(s) \geq 0$; thus we can extend the $s$-domain to the nonnegative $s$-plane. Also, $\mathcal{P}$ diverges at most like a power in $A D_{\theta, c}$, and hence the $\lambda$ integral is bounded due to the presence of the $\mathrm{e}^{-\lambda t}$ $(t>0)$; eventually, the $t$ integral is also bounded by the same factor for all $s$. This means that the second term is the product of $\frac{s}{\Gamma(s)}$ with a function $r_{1}(s)$ that is regular for all $\operatorname{Re}(s) \geq 0$. For the first term, we need to rewrite the contour as $\Lambda_{\theta, c}=\Lambda_{\theta,-c}-C_{c}$. In the integral on the new contour $\Lambda_{\theta,-c}$ we can use the expansion of $\mathcal{P}$; the other gives

$$
\frac{1}{2 \pi i} \int_{C_{c}} \frac{\mathrm{e}^{-\lambda t}}{-\lambda} \mathcal{P}(s, \lambda, S, U) d \lambda=\mathcal{P}(s, 0, S, U)=0,
$$

since $\mathcal{T}(s, \lambda, S, U)=0$ because $\log \Gamma\left(0, S_{n}\right)=0$ by definition, $P_{\rho_{l}}(0)=0$, and $\phi_{\sigma_{h}}(0)=0$ since $\lambda=0$ belongs to $A D_{\theta, c}$. We are left with

$$
\zeta_{1}(s)=\frac{s}{\Gamma(s)} \int_{0}^{1} t^{s-1} \frac{1}{2 \pi i} \int_{\Lambda_{\theta,-c}} \frac{\mathrm{e}^{-\lambda t}}{-\lambda} \mathcal{P}(s, \lambda, S, U) d \lambda d t,
$$

where we can use the expansion of $\mathcal{P}$, given in Lemma 3.5. We obtain (use equations (13) and (14) in the appendix)

$$
\begin{aligned}
\zeta_{1}(s)= & -\frac{s}{\Gamma(s)} \sum_{j=0}^{N} A_{\alpha_{j}, 0}(s) \frac{1}{s-\alpha_{j}} \frac{1}{\Gamma\left(1-\alpha_{j}\right)}-\frac{s}{\Gamma(s)} \sum_{j=0}^{1} A_{j, 1}(s) \frac{1}{s-j} \frac{\psi(1-j)}{\Gamma(1-j)} \\
& -\frac{s}{\Gamma(s)} \sum_{j=0}^{1} A_{j, 1}(s) \frac{1}{(s-j)^{2}} \frac{1}{\Gamma(1-j)}+\frac{s}{\Gamma(s)} r_{2}(s),
\end{aligned}
$$


with $r_{2}(s)$ regular near $s=0$. Since by definition and Lemma 3.3, $\alpha_{N} \leq 0$, near $s=0$ the only nonvanishing terms are those with $\alpha_{j}=0$; hence

$$
\zeta_{1}(s)=\frac{s}{\Gamma(s)}\left(-\frac{1}{s} A_{0,0}(s)+\frac{1}{s} \gamma A_{0,1}(s)-\frac{1}{s^{2}} A_{0,1}(s)+r_{3}(s)\right),
$$

where $r_{3}(s)$ is regular near $s=0$. Summing up we have the thesis.

Lemma 3.7. The functions $\Phi_{\sigma_{h}}(s)$ have at most a double pole at $s=0$.

Proof. Just proceed as in the proof of Lemma 3.6 and use the asymptotic expansion given for $\phi_{\sigma_{h}}$ in Lemma 3.3 .

Remark 3.8. Note that $\zeta_{2}(s)$ can have at most a simple pole at $s=0$. In fact, by definition, $\zeta(s, U)$ has at most simple poles. On the other side, $\Phi_{\sigma_{h}}(s)$ also has at most double poles, by the previous lemma, and this implies the statement, since $\frac{s}{\Gamma(s)}$ has a zero of order 2 at $s=0$. Note also that we cannot use the expansion of $\phi_{\sigma_{h}}(\lambda)$ to compute the derivative of $\zeta_{2}(s)$ at $s=0$, since the remainder of such an expansion would appear in $\zeta_{2}^{\prime}(0)$. We need explicit computation of the integral defining the function $\Phi_{\sigma_{h}}(s)$ and an expansion of $\Phi_{\sigma_{h}}(s)$ near $s=0$ at least up to the constant term.

We can now state and prove our main results in the following theorem.

Theorem 3.9 (Spectral decomposition lemma). Let the double sequence $S$ be spectrally decomposable over $U$ with power $\kappa$ and length $\ell$. Then

$$
\begin{aligned}
\operatorname{Res}_{s=0} \zeta(s, S)= & \frac{1}{\kappa} \sum_{h=0}^{\ell} \operatorname{Res}_{s=0} \Phi_{\sigma_{h}}(s) \operatorname{Res}_{s=\sigma_{h}} \zeta(s, U), \\
\operatorname{Res}_{s=0} \zeta(s, S)= & \sum_{h=0}^{\ell} \operatorname{Res}_{s=0} \Phi_{\sigma_{h}}(s) \operatorname{Res}_{s=\sigma_{h}} \zeta(s, U)-A_{0,1}(0) \\
& +\frac{1}{\kappa} \sum_{h=0}^{\ell} \operatorname{Res}_{s=\sigma_{h}} \zeta(s, U)\left(\operatorname{Res}_{s=0} \Phi_{\sigma_{h}}(s)+\gamma \operatorname{Res}_{s=0} \Phi_{\sigma_{h}}(s)\right), \\
\operatorname{Res}_{s=0} \zeta^{\prime}(s, S)= & \frac{1}{\kappa}\left(\frac{\gamma^{2}}{2}-\frac{\pi^{2}}{12}\right) \sum_{h=0}^{\ell} \operatorname{Res}_{s=0} \Phi_{\sigma_{h}}(s) \operatorname{Res}_{s=\sigma_{h}} \zeta(s, U) \\
& +\frac{\gamma}{\kappa} \sum_{h=0}^{\ell} \operatorname{Res}_{s=0} \Phi_{\sigma_{h}}(s) \operatorname{Res}_{s=\sigma_{h}} \zeta(s, U)+\gamma \sum_{h=0}^{\ell} \operatorname{Res}_{s=0} \Phi_{\sigma_{h}}(s) \operatorname{Res}_{s=\sigma_{h}} \zeta(s, U) \\
& +\frac{1}{\kappa} \sum_{h=0}^{\ell} \operatorname{Res}_{s=0} \Phi_{\sigma_{h}}(s) \operatorname{Res}_{s=\sigma_{h}} \zeta(s, U)+\kappa \sum_{h=0}^{\ell} \operatorname{Res}_{s=0} \Phi_{\sigma_{h}}(s) \operatorname{Res}_{s=\sigma_{h}} \zeta^{\prime}(s, U) \\
& +\sum_{h=0}^{\ell} \operatorname{Res}_{s=0} \Phi_{\sigma_{h}}(s) \operatorname{Res}_{s=\sigma_{h}} \zeta(s, U)-A_{0,0}(0)-A_{0,1}^{\prime}(0) .
\end{aligned}
$$

Proof. Let $s$ be in a small neighborhood of $s=0$. Then, by Lemma 3.6.

$$
\zeta(s, S)=\frac{1}{\Gamma(s)}\left(\gamma A_{0,1}(s)-A_{0,0}(s)-\frac{A_{0,1}(s)}{s}+s \sum_{h=0}^{\ell} \Phi_{\sigma_{h}}(s) \zeta\left(\kappa s+\sigma_{h}, U\right)+s r(s)\right),
$$


where $r(s)$ is regular. We are able to expand $\zeta(s, S)$ near $s=0$, using the expansions of the single factors provided by the previous results. By Lemma 3.5 the functions $A_{i, j}(s)$ are regular near $s=0$. By Definition 3.1, the function $\zeta(s, U)$ has at most simple poles, and therefore we have the expansion

$$
\zeta\left(\kappa s+\sigma_{h}, U\right)=\frac{1}{\kappa} \operatorname{Res}_{s=\sigma_{h}} \zeta(s, U) \frac{1}{s}+\operatorname{Res}_{s=\sigma_{h}} \zeta(s, U)+\kappa \operatorname{Res}_{s=\sigma_{h}} \zeta^{\prime}(s, U) s+O\left(s^{2}\right),
$$

near $s=0$. By Lemma 3.7 and Remark 3.8, the functions $\Phi_{\sigma_{h}}(s)$ have at most a pole of order 2 at $s=0$, and therefore we have an expansion of the form

$$
\Phi_{\sigma_{h}}(s)=\frac{\operatorname{Res}_{2 s=0} \Phi_{\sigma_{h}}(s)}{s^{2}}+\frac{\operatorname{Res}_{1 s=0} \Phi_{\sigma_{h}}(s)}{s}+\operatorname{Res}_{s=0} \Phi_{\sigma_{h}}(s)+O(s) .
$$

Substituting these expansions and the classical expansion of $1 / \Gamma(s)$ in equation (12), after some calculations, we obtain the thesis.

\section{APPENDIX}

We give in this appendix explicit formulas for some tricky contour integrals appearing in the text (see 13 for proofs). Here $a$ is any real number.

$$
\begin{gathered}
\frac{1}{2 \pi i} \int_{\Lambda_{\theta,-c}} \mathrm{e}^{-\lambda}(-\lambda)^{a} d \lambda=-\frac{1}{\Gamma(-a)} \\
\frac{1}{2 \pi i} \int_{\Lambda_{\theta,-c}} \mathrm{e}^{-\lambda}(-\lambda)^{a} \log (-\lambda) d \lambda=-\frac{\psi(-a)}{\Gamma(-a)} \\
\int_{0}^{\infty} t^{s-1} \frac{1}{2 \pi i} \int_{\Lambda_{\theta, c}} \frac{\mathrm{e}^{-\lambda t}}{-\lambda} \frac{1}{(1-\lambda)^{a}} d \lambda d t=\frac{\Gamma(s+a)}{\Gamma(a) s} \\
\int_{0}^{\infty} t^{s-1} \frac{1}{2 \pi i} \int_{\Lambda_{\theta, c}} \frac{\mathrm{e}^{-\lambda t} \frac{\log (1-\lambda)}{-\lambda} d \lambda d t=\frac{\Gamma(s+a)}{\Gamma(a) s}(\psi(a)-\psi(s+a)),}{(1-\lambda)^{a}} d \frac{1}{2 \sqrt{\pi}} \frac{\Gamma\left(s+\frac{1}{2}\right)}{s^{2}} .
\end{gathered}
$$

\section{REFERENCES}

[1] J. Brüning and R. Seeley, The resolvent expansion for second order regular singular operators, J. Funct. Anal. 73 (1988) 369-415. MR899656 (88g:35151)

[2] J. Choi and J.R. Quine, Zeta regularized products and functional determinants on spheres, Rocky Mount. Jour. Math. 26 (1996) 719-729. MR1406503(97k:58176)

[3] P.B. Gilkey, Invariance theorems, the heat equation, and the Atiyah-Singer index theorem (Studies in Adv. Math., CRC Press, 1995). MR1396308 (98b:58156)

[4] G.H. Hardy and M. Riesz, The general theory of Dirichlet series, Cambridge University Press, 1915.

[5] J. Jorgenson and S. Lang, Basic analysis of regularized series and products, Lect. Notes in Math. 1564, Springer-Verlag, 1993. MR1284924 (95e:11094)

[6] J. Jorgenson and S. Lang, Explicit formulas for regularized products and series, Lect. Notes in Math. 1593, Springer-Verlag, 1994. MR.1329730 (96f:11110)

[7] J. Jorgenson and S. Lang, Artin formalism and heat kernels, J. Reine Angew. Math. 447 (1994) 165-200. MR.1263173 (95c:11106)

[8] J. Jorgenson and S. Lang, On Cramér's theorem for general Euler products with functional equations, Math. Ann. 297 (1993) 383-416. MR1245398 (94k:11101)

[9] J. D. Murray, Asymptotic analysis, Springer-Verlag, 1984. MR740864 (85m:34085)

[10] J. R. Quine, S. H. Heydari and R. Y. Song, Zeta regularized products, Trans. Amer. Math. Soc. 338 (1993) 213-230. MR.1100699 (93j:58139) 
[11] D. B. Ray and I. M. Singer, R-torsion and the Laplacian on Riemannian manifolds, Adv. Math. 7 (1974) 145-210. MR0295381 (45:4447)

[12] M. Spreafico, A generalization of the Euler gamma function, Funct. Anal. Appl. 39 (2005) 87-91. MR 2161522 (2006j:33005)

[13] M. Spreafico, Zeta function and regularized determinant on a disc and on a cone, J. Geom. Phys. 54 (2005) 355-371. MR2139088 (2005k:11184)

[14] M. Spreafico, Zeta invariants for sequences of spectral type, special functions and the Lerch formula, Proc. Royal Soc. Edinburgh. 281 (2006) 865-889. MR2250451 (2007e:11108)

[15] M. Spreafico, Zeta invariants for Dirichlet series, Pacific J. Math. 274 (2006) 185-199. MR:2231657 (2007b:11129)

[16] M. Spreafico and S. Zerbini, Spectral analysis and zeta determinants on the deformed spheres, Comm. Math. Phys. 273 (2007) 677-704. MR2318862 (2008h:58063)

[17] A. Voros, Spectral functions, special functions and the Selberg zeta function, Comm. Math. Phys. 110 (1987) 439-465. MR.891947 (89b:58173)

ICMC, Universidade São Paulo, São Carlos, 13556-560 Brazil

E-mail address: mauros@icmc.usp.br 